\title{
Acute kidney injury in hospitalized patients with nonexacerbated chronic obstructive pulmonary disease
}

\author{
Xiaohong Wang ${ }^{1}$, Zhen Xie $\mathrm{i}^{2,3^{*}}$ (D), Shuguang Xiong ${ }^{1}$, Wei Xiong ${ }^{3,4}$, Tian Zhong ${ }^{3,5}$ and Yang $\mathrm{Su}^{3,6^{*}}$
}

\begin{abstract}
Background: The epidemiology of acute kidney injury (AKI) in nonexacerbated chronic obstructive pulmonary disease (NECOPD) patients is unknown. This study investigated the factors associated with AKI and the association between AKI and in-hospital mortality in the hospitalized NECOPD population.

Methods: The electronic medical records of 2897 patients hospitalized with NECOPD were analyzed retrospectively. Demographic information, medicine used before AKI, diagnosis records and laboratory data were collected. AKI was classified as community-acquired (CA-) or hospital-acquired (HA-) AKI according to the serum creatinine criteria. Risk factors for HA-AKI and in-hospital mortality were analyzed by logistic regression analyses. To avoid an interaction between cor pulmonale and AKI, the association between AKI and in-hospital morality was further analyzed with cor pulmonale stratification.

Results: The incidence rates of CA- and HA-AKI were 7.1 and 12.0\%, respectively. Increased age, female sex, cor pulmonale comorbidity, chronic kidney disease stage, diuretic and glycopeptide use before AKI and iodinecontaining contrast medium exposure were independently associated with HA-AKI. A total of $5.7 \%$ of the patients died. After adjustment for age, sex, cor pulmonale, chronic kidney disease, Charlson comorbidity index score (without renal disease) and hemoglobin level, HA-AKI was an independent risk factor for in-hospital mortality [OR 13.909 (95\% Cl 8.699-22.238) in non-cor pulmonale subgroup; OR 26.604 (95\% Cl 12.166-58.176) in cor pulmonale subgroup], whereas CA-AKI was not.
\end{abstract}

Conclusions: AKI is common in the NECOPD population. Diuretics and contrast media are associated with HA-AKI in this population. The patients with HA-AKI have a higher mortality risk than the patients without AKI.

Keywords: Nonexacerbated chronic obstructive pulmonary disease, Acute kidney injury, Epidemiological study, Risk factor, Clinical burden, In-hospital mortality

\footnotetext{
*Correspondence: xiezhen6289@126.com; 19822716@qq.com

${ }^{2}$ Department of Dermatology, Sichuan Provincial People's Hospital, University

of Electronic Science and Technology of China, Chengdu, China

${ }^{3}$ Chinese Academy of Sciences Sichuan Translational Medicine Research

Hospital, 32 West Second Section, First Ring Road, Chengdu 610031, China

${ }^{6}$ Clinical Laboratory, Sichuan Provincial People's Hospital, University of

Electronic Science and Technology of China, Chengdu, China

Full list of author information is available at the end of the article
}

C C The Author(s). 2020 Open Access This article is licensed under a Creative Commons Attribution 4.0 International License, which permits use, sharing, adaptation, distribution and reproduction in any medium or format, as long as you give appropriate credit to the original author(s) and the source, provide a link to the Creative Commons licence, and indicate if changes were made. The images or other third party material in this article are included in the article's Creative Commons. licence, unless indicated otherwise in a credit line to the material. If material is not included in the article's Creative Commons licence and your intended use is not permitted by statutory regulation or exceeds the permitted use, you will need to obtain permission directly from the copyright holder. To view a copy of this licence, visit http://creativecommons.org/licenses/by/4.0/ The Creative Commons Public Domain Dedication waiver (http://creativecommons.org/publicdomain/zero/1.0/) applies to the data made available in this article, unless otherwise stated in a credit line to the data. 


\section{Background}

Chronic obstructive pulmonary disease (COPD) is characterized by chronic airflow limitation, inflammation and lung remodeling. It is a progressive disease that results in a significant burden, both medically and financially. COPD is the third leading cause of death among diseases, resulting in a great economic burdens to society [1]. Acute exacerbations of COPD (AECOPD) require emergent medical care, and their estimated 3-year all-cause mortality rates are as high as $50 \%$ in patients who require hospitalization [2]. In addition to urgent therapy for AECOPD, care under stable conditions is also essential for COPD patients. It is worth noting that many hospitalized patients admitted to respiratory departments or other units have nonexacerbated COPD (NECOPD). Even without respiratory infection, the patients with NECOPD had a relatively higher risk of mortality [3]. Hypoxemia is an elementary pathophysiological disorder associated with COPD, regardless of acute or stable conditions. Due to the systemic damage of hypoxemia, the recognition of extrapulmonary complications will facilitate effective personal management.

The lung interacts with the kidney in the body [4]. Acute kidney injury (AKI) is a common syndrome in clinical practice. According to a preliminary analysis of 266 studies worldwide, $21 \%$ of hospitalized patients presented AKI [5]. AKI is closely associated with a high clinical burden and poor prognosis, such as in-hospital mortality, and therefore is considered a high-risk complication. Previous studies have shown that AKI increases in-hospital mortality in patients with AECOPD $[6,7]$. Fabbian et al. [6] reported a 5\% AKI incidence in 7073 patients. They also demonstrated that AKI was associated with a $284.9 \%$ higher mortality risk. They used the International Classification of Diseases codes, which were specific but had low sensitivity, as an AKI diagnosis standard [8]. Recently, Cao et al. [7] reported an overall AKI prevalence of $21.3 \%$ in the AECOPD population using the Kidney Disease: Improving Global Outcomes (KDIGO) criteria. Notably, although all the abovementioned studies investigated the risk factors of AKI, they did not consider the influence of drug use.

Compared with AECOPD, NECOPD has different pathophysiological disturbances and clinical features. Patients with AECOPD are hospitalized mainly in a respiratory department or intensive care unit, as a high proportion of patients require pulmonary support, whereas NECOPD patients may be admitted to other divisions. Furthermore, the complications, medications and diagnostic techniques for AECOPD and NECOPD differ slightly. Although NECOPD has been included as a variable in the analysis of risk factors, such as the Cleveland scoring system to predict AKI after cardiac surgery for AKI [9], the epidemiology of
AKI in patients with NECOPD has not yet been investigated.

In this study, we investigated the factors associated with AKI and the association between AKI and inhospital mortality in the hospitalized NECOPD population.

\section{Methods \\ Study patients}

The current study retrospectively analyzed the clinical data of adult COPD patients ( $\geq 18$ years) who received treatment at Sichuan Provincial People's Hospital between January 2011 and September 2016. All the patients with COPD discharge diagnoses were selected. The patients with a diagnosis of AECOPD or any comorbid respiratory infection were considered to have AECOPD, while the others were considered to have NECOPD. The exclusion criteria were as follows: 1) the patient's baseline serum creatinine levels were unavailable; 2) the patient had advanced chronic kidney disease (CKD), i.e., an estimated glomerular filtration rate (eGFR) $<15 \mathrm{ml} / \mathrm{min} / 1.73 \mathrm{~m}^{2}$; a minimum $\mathrm{SCr}$ level $>$ $353.6 \mu \mathrm{mol} / \mathrm{L}(4 \mathrm{mg} / \mathrm{dL})$ during the hospital stay; a clinical diagnosis of stage $5 \mathrm{CKD}$; or the receipt of a replacement kidney before the onset of AKI; 3) the patient had a maximum $\mathrm{SCr}$ level $<53 \mu \mathrm{mol} / \mathrm{L}$ during the hospital stay (to avoid an AKI diagnosis due to a very slight $\mathrm{SCr}$ increase and potential malnutrition); 4) the patient previously underwent kidney transplantation or amputation; and 5) the patient had incomplete clinical records that were unable to supply the data for the current research.

Based on the clinical records, medical costs, lengths of hospital stay and in-hospital mortality rates associated with AKI were analyzed as medical burdens; the main outcome was in-hospital mortality.

\section{Observational indices}

AKI was diagnosed according to the criteria provided by the Kidney Disease: Improving Global Outcomes (KDIGO) guidelines; AKI was confirmed if the $\mathrm{SCr}$ value increased by more than $50 \%$ compared to the baseline value within 7 days [10]. Considering that the $\mathrm{SCr}$ tests before admission are frequently not available, the lowest creatinine level from 1 month before admission to the time of discharge was regarded as the baseline for community-acquired (CA-) AKI. Patients admitted to the hospital with AKI based on a creatinine measurement within $48 \mathrm{~h}$ of hospital admission were considered to have CA-AKI [11]; the other patients were considered to have hospital-acquired (HA-) AKI [10]. CKD was classified based on the eGFR, which was calculated by the minimal $\mathrm{SCr}$ value within 1 year, according to the EPI equation of the Chronic Kidney Disease Epidemiology Collaboration [12]. 
Complications were adjusted for based on discharge diagnoses, and the Charlson comorbidity index (CCI) score was evaluated (the $\mathrm{CCI}$ is a scoring system that assigns weights to mortality-related comorbid conditions, and it involves 19 comorbidities, namely, myocardial infarction, congestive heart failure, peripheral disease, cerebrovascular disease, chronic pulmonary disease, dementia, connective tissue disease, peptic ulcer disease, mild liver disease, diabetes without end-organ damage, hemiplegia, mild to moderate renal disease, diabetes with end-organ damage, tumor without metastasis, leukemia, lymphoma, moderate or severe liver disease and metastatic solid tumor) [13]. The total CCI score was used as the primary index for predicting in-hospital mortality [14]. The renal disease item in the CCI was assessed based on either a corresponding diagnosis of moderate to severe renal disease or an eGFR $<45 \mathrm{ml} / \mathrm{min} / 1.73 \mathrm{~m}^{2}$. We also calculated the CCI score after removing renal disease to avoid potential collinearity between CKD and $\mathrm{CCI}$ when they were both included as variables in one multivariable analysis. Anemia was defined as a minimum hemoglobin $(\mathrm{Hb})$ concentration $<120 \mathrm{~g} / \mathrm{L}$ during hospitalization, regardless of sex, according to the World Health Organization standard, because amenorrhea data was unavailable in the female subjects. The $\mathrm{Hb}$ level was stratified as $<6 \mathrm{~g} / \mathrm{dL}, 6-8.9 \mathrm{~g} /$ $\mathrm{dL}, 9-11.9 \mathrm{~g} / \mathrm{dL}$ and $\geq 12 \mathrm{~g} / \mathrm{dL}$.

\section{Statistical analyses}

Measurement data are presented as the median [25th quartile (Q25)-75th quartile Q75)], and independent Mann-Whitney-U tests were performed for group comparisons. Enumeration data are presented as case numbers, and $\chi^{2}$ tests were used for the comparisons. Multiple comparisons were corrected using the Bonferroni method. Univariate logistic regression was performed, and the variables with a statistically significant difference were included in the multivariate logistic regression analysis by a stepwise forward conditional model. Odds ratios (ORs) and 95\% confidence intervals (CIs) were calculated. All statistical analyses were performed with SPSS 24.0, and a bilateral $P$ value $<0.05$ was considered statistically significant.

\section{Results}

\section{General data}

A total of 11,199 patients hospitalized for COPD were investigated during this study, 4898 patients were nonacute. After applying the exclusion criteria, this study enrolled 2897 NECOPD patients. Compared with the excluded patients, those enrolled were 1 year older (75 vs $74, P=0.003$ ), more often male $(77.9 \%$ vs $67.2 \%, P<0.001)$, less often had cor pulmonale $(22.8 \%$ vs $55.9 \%, P<0.001)$, and had an eGFR that was $4.7 \mathrm{ml} / \mathrm{min} / 1.73 \mathrm{~m}^{2}$ lower $(91.7$ vs 87.0, $P<0.001)$. Among these patients, 205 patients (7.1\%) had CA-AKI, and 349 patients (12.0\%) had HA-AKI (Fig. 1). In the CA-AKI and HA-AKI groups, $62(9.4 \%)$ and $113(17.1 \%)$ patients, respectively, presented with cor pulmonale complications. The general data of the patients with the different types of AKI are summarized in Table 1.

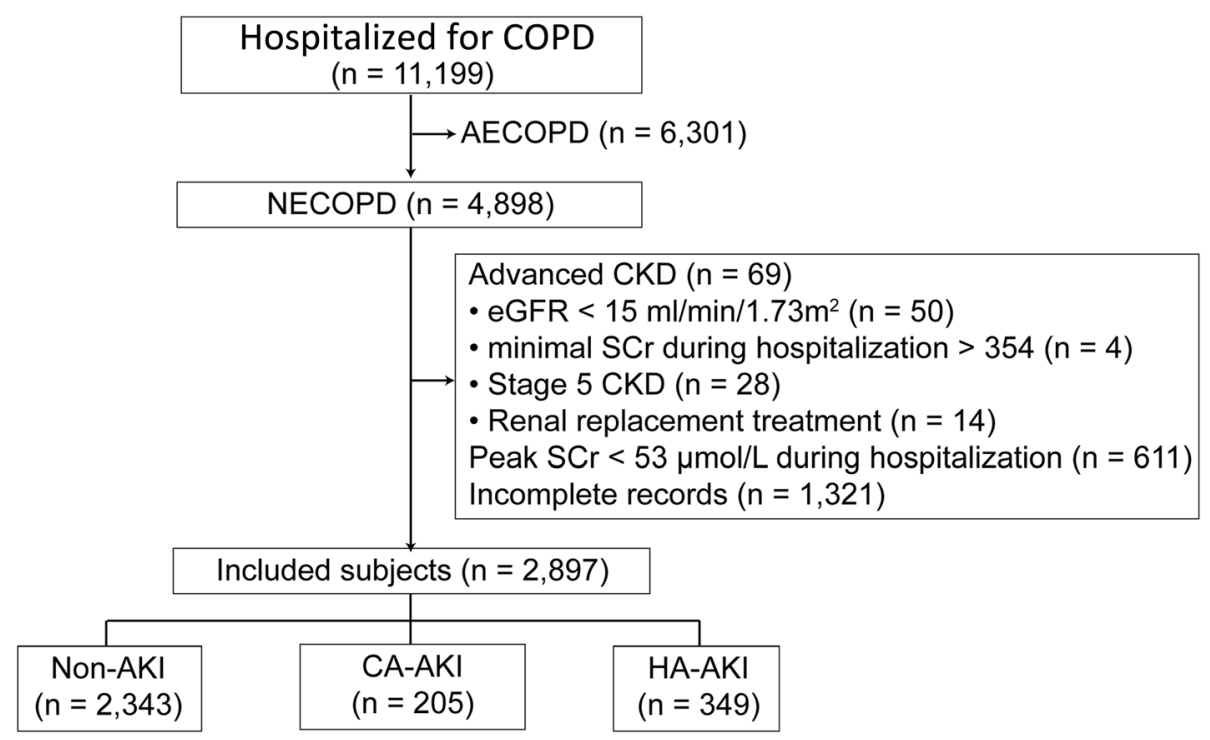

Fig. 1 Inclusion flow chart. COPD: chronic obstructive pulmonary disease; NECOPD: nonexacerbated COPD; CKD: chronic kidney disease; eGFR: estimated glomerular filtration rate; SCr: serum creatinine; AKI: acute kidney injury; CA: community-acquired; HA: hospital-acquired 
Table 1 The basic clinical characteristics of the study patients

\begin{tabular}{|c|c|c|c|c|}
\hline & Non-AKI & CA-AKI & HA-AKI & $P$ value \\
\hline Age, year & $75(67,82)$ & $74(66,82)$ & $78(72,84)$ & $<0.001$ \\
\hline Male, $n(\%)$ & $1852(79.0 \%)$ & $158(77.1 \%)$ & $247(70.8 \%)$ & 0.002 \\
\hline Cor pulmonale comorbidity, $n$ (\%) & $486(20.7 \%)$ & $62(30.2 \%)$ & $113(32.4 \%)$ & $<0.001$ \\
\hline Admission department, $n$ (\%) & & & & $<0.001$ \\
\hline Respiratory & $576(24.6 \%)$ & $54(26.3 \%)$ & $82(23.5 \%)$ & \\
\hline Intensive care & $134(5.7 \%)$ & $21(10.2 \%)$ & $44(12.6 \%)$ & \\
\hline Cardiovascular & $426(18.2 \%)$ & $27(13.2 \%)$ & $80(22.9 \%)$ & \\
\hline Other & $1207(51.5 \%)$ & $103(50.2 \%)$ & $143(41.0 \%)$ & \\
\hline Maximal SCr $(\mu \mathrm{mol} / \mathrm{L})$ & $92.4 \pm 31.4$ & $115.0 \pm 57.6$ & $176.3 \pm 117.4$ & $<0.001$ \\
\hline Minimal SCr $(\mu \mathrm{mol} / \mathrm{L})$ & $78.4 \pm 25.9$ & $67.5 \pm 25.4$ & $88.4 \pm 46.2$ & $<0.001$ \\
\hline $\mathrm{eGFR}\left(\mathrm{ml} / \mathrm{min} / 1.73 \mathrm{~m}^{2}\right)$ & $87.1 \pm 18.3$ & $96.6 \pm 23.6$ & $80.2 \pm 27.8$ & $<0.001$ \\
\hline Anemia, $n(\%)$ & $1173(50.1 \%)$ & $135(65.9 \%)$ & $267(76.5 \%)$ & $<0.001$ \\
\hline $\mathrm{CCl}$ & $5(3,6)$ & $5(3,7)$ & $5(4,8)$ & 0.148 \\
\hline
\end{tabular}

SCr serum creatinine; eGFR estimated glomerular filtration rate; $\mathrm{CCl}$ Charlson comorbidity index

\section{Factors associated with HA-AKI}

Due to a lack of preadmission data for the CA-AKI group, only the associations between clinical data and the development of HA-AKI were analyzed in this study. As medication concerned sensitive data, only drugs that are commonly used for diseases of respiratory origin with potential renal toxicity were retrieved in this study. The prescription time and AKI occurrence were analyzed, and only the medicines used before AKI were further studied. These drugs included proton pump inhibitors (use prevalence, 55.0\%), diuretics (including osmotic diuretics such as mannitol; use prevalence, 28.6\%), rennin-angiotensin system inhibitors (use prevalence, 26.7\%), nonsteroidal anti-inflammatory drugs (NSAIDs; use prevalence, $18.8 \%$ ), intravenous diodone (use prevalence, 12.2\%), intravenous antihemorrhagic drugs (use prevalence, $10.0 \%$ ), aminoglycosides (use prevalence, $4.8 \%$ ) and glycopeptides (use prevalence, 2.4\%).

Table 2 Analysis of the risk factors for HA-AKI

\begin{tabular}{|c|c|c|c|c|c|c|}
\hline & \multicolumn{3}{|c|}{ Univariate model } & \multicolumn{3}{|c|}{ Multivariate model } \\
\hline & $\mathrm{OR}$ & $95 \% \mathrm{Cl}$ & $P$ value & $\mathrm{OR}$ & $95 \% \mathrm{Cl}$ & $P$ value \\
\hline Increased age & 1.899 & $1.324-2.724$ & 0.001 & 1.463 & $1.003-2.134$ & 0.048 \\
\hline Female & 1.558 & $1.212-2.002$ & 0.001 & 1.498 & $1.149-1.952$ & 0.003 \\
\hline Cor pulmonale & 1.830 & $1.431-2.339$ & $<0.001$ & 1.790 & $1.379-2.324$ & $<0.001$ \\
\hline CKD & & & $<0.001$ & & & $<0.001$ \\
\hline Stage $1-2$ & Reference & & & Reference & & \\
\hline Stage $3 a$ & 1.775 & $1.163-2.708$ & 0.008 & 1.754 & $1.136-2.709$ & 0.011 \\
\hline Stage 3b & 3.139 & $1.862-5.292$ & $<0.001$ & 3.161 & $1.850-5.401$ & $<0.001$ \\
\hline Stage 4 & 6.478 & $3.532-11.882$ & $<0.001$ & 6.575 & $3.531-12.243$ & $<0.001$ \\
\hline Diabetes & 1.357 & $1.045-1.762$ & 0.022 & Not entered in & model & \\
\hline \multicolumn{7}{|l|}{ Clinical medication } \\
\hline Proton pump inhibitors & 1.142 & $0.910-1.434$ & 0.253 & Not selected & & \\
\hline Diuretics/dehydrants & 1.950 & $1.547-2.459$ & $<0.001$ & 1.527 & $1.193-1.954$ & 0.001 \\
\hline RAS inhibitors & 1.081 & $0.841-1.389$ & 0.544 & Not selected & & \\
\hline NSAIDs & 0.967 & $0.723-1.293$ & 0.821 & Not selected & & \\
\hline Intravenous diodones & 1.928 & $1.436-2.588$ & $<0.001$ & 2.048 & $1.499-2.798$ & $<0.001$ \\
\hline Intravenous hemostatics & 0.927 & $0.632-1.359$ & 0.697 & Not selected & & \\
\hline Aminoglycosides & 1.256 & $0.769-2.053$ & 0.362 & Not selected & & \\
\hline Glycopeptides & 4.141 & $2.469-6.944$ & $<0.001$ & 3.991 & $2.322-6.859$ & $<0.001$ \\
\hline
\end{tabular}

HA-AKI hospital acquired-acute kidney injury; CKD chronic kidney disease; RAS renin-angiotensin system; NSAIDs nonsteroidal anti-inflammatory drugs 
Univariate regression showed that increased age, female gender, cor pulmonale complication, CKD and diabetes were associated with HA-AKI. Among the drugs with a use prevalence greater than $10 \%$, diuretics and intravenous diodones used before AKI were associated with HAAKI. Despite a low use prevalence, glycopeptides were associated with the development of HA-AKI (Table 2). The variables with $P$ values $<0.10$, i.e., age $>65$ years, female gender, diabetes complication, cor pulmonale complication, CKD stage, and pre-AKI diuretic, diodone and glycopeptide use, were included in the multivariate model, according to the univariate regression analyses (Table 2).

\section{AKI-related clinical burdens}

The total hospitalization costs for the non-, CA- and HA-AKI groups were 3238 (2216, 5003), 4484 (3165, $7756)$ and $4759(3028,7481)$ US dollars, with daily costs of $220(168,312), 279(205,480)$ and $308(213,518)$ US dollars, respectively; the lengths of hospital stay for the three groups were 14 days $(11,20), 16$ days $(13,21)$ and 16 days $(13,22)$, respectively (the data regarding the costs and hospital stays are all presented as the median (Q25, Q75); therefore, the median of the total hospitalization costs cannot be obtained by multiplying the median of the daily costs with that of the hospital stays in the corresponding group); there were significant differences between the AKI groups and non-AKI group $(P<0.001)$.

A total of 164 patients (5.7\%) died during hospitalization. Compared with the surviving patients, the nonsurviving patients were older, more likely be female and had higher comorbidity burdens (Table 3). The relation between AKI and in-hospital mortality was further analyzed. According to the univariate regression

Table 3 The basic clinical characteristics of surviving and nonsurviving patients

\begin{tabular}{llll}
\hline & Surviving & Nonsurviving & $P$ value \\
\hline Age, years & $74 \pm 10$ & $79 \pm 8$ & $<0.001$ \\
Male, $n(\%)$ & $2142(78.4 \%)$ & $115(70.1 \%)$ & 0.013 \\
Cor pulmonale comorbidity, $n(\%)$ & $603(22.1 \%)$ & $58(35.4 \%)$ & $<0.001$ \\
Admission department, $n(\%)$ & & & $<0.001$ \\
$\quad$ Respiratory & $657(24.0 \%)$ & $55(33.5 \%)$ & \\
$\quad$ Intensive care & $167(6.1 \%)$ & $32(19.5 \%)$ & \\
Cardiovascular & $510(18.7 \%)$ & $23(14.0 \%)$ & \\
Other & $1399(51.2 \%)$ & $54(32.9 \%)$ & \\
eGFR (ml/min/1.73 m $\left.{ }^{2}\right)$ & $87.4 \pm 19.9$ & $80.3 \pm 26.7$ & $<0.001$ \\
Anemia, $n$ (\%) & $1447(52.9 \%)$ & $128(78.0 \%)$ & $<0.001$ \\
CCI score & $3(2,4)$ & $4(2,8)$ & $<0.001$ \\
\hline $\begin{array}{l}\text { SCr serum creatinine; eGFR estimated glomerular filtration rate; CCl Charlson } \\
\text { comorbidity index }\end{array}$ & &
\end{tabular}

analysis, age $>65$ years, female gender, cor pulmonale complication, CKD stage, CCI score without renal disease, $\mathrm{Hb}$ level (according to the minimum values during hospital stay, $<6 \mathrm{~g} / \mathrm{dL}, 6-8.9 \mathrm{~g} / \mathrm{dL}, 9-11.9 \mathrm{~g} / \mathrm{dL}$ and $\geq 12$ $\mathrm{g} / \mathrm{dL}$ ) and AKI type were associated with in-hospital mortality (Table 4). A multivariate logistic regression model was then constructed for further analysis. Because the CCI already considers CKD, the CCI score without renal disease but not the CCI score with renal disease was included in the model. In addition, due to the interaction between AKI and cor pulmonale according to the preliminary analysis, the cor pulmonale variable was excluded from the multivariate model. The results showed an independent association of in-hospital mortality with increased age [OR 2.051 (95\% CI 1.305-3.591)], decreased $\mathrm{Hb}$ [OR 1.140 (95\% CI 0.727-1.786) for 9-11.9 $\mathrm{g} / \mathrm{dL}$; OR 2.165 (95\% CI $1.305-3.591$ ) for $6-8.9 \mathrm{~g} / \mathrm{dL}$; OR 6.403 (95\% CI 2.986-13.730) for $<6 \mathrm{~g} / \mathrm{dL}$ ] and AKI [OR 2.186 (95\% CI 1.072-4.455) for CA-AKI; OR 16.949 (95\% CI 11.483-25.016) for HA-AKI] (Fig. 2a). Then, we analyzed the independent relation between AKI and inhospital mortality stratified by cor pulmonale. In both of the subgroups (with or without cor pulmonale), only HA-AKI was an independent risk factor for in-hospital mortality [OR 13.909 (95\% CI 8.699-22.238) in the noncor pulmonale subgroup; OR 26.604 (95\% CI $12.166-$

Table 4 Analytical outcomes of AKI and in-hospital mortality according to the univariate model

\begin{tabular}{|c|c|c|c|}
\hline & OR & 95\% confidence interval & $P$ \\
\hline Increased age & 3.046 & $1.639-5.660$ & $<0.001$ \\
\hline Female & 1.544 & $1.092-2.184$ & 0.014 \\
\hline Cor pulmonale & 1.933 & $1.386-2.696$ & $<0.001$ \\
\hline CKD & & & $<0.001$ \\
\hline Stage 1-2 & Reference & & \\
\hline Stage $3 a$ & 1.351 & $0.715-2.554$ & 0.354 \\
\hline Stage 3b & 2.382 & $1.165-4.872$ & 0.017 \\
\hline Stage 4 & 6.834 & $3.450-13.539$ & $<0.001$ \\
\hline Diabetes & 1.170 & $0.805-1.702$ & 0.411 \\
\hline CCl score & 1.189 & $1.134-1.247$ & $<0.001$ \\
\hline In-hospital minimum $\mathrm{Hb}$ & & & $<0.001$ \\
\hline$\geq 120 \mathrm{~g} / \mathrm{L}$ & Reference & & \\
\hline $90-119$ & 2.134 & $1.419-3.210$ & $<0.001$ \\
\hline $60-89$ & 5.456 & $3.486-8.538$ & $<0.001$ \\
\hline $30-59$ & 10.058 & $5.205-19.436$ & $<0.001$ \\
\hline \multicolumn{4}{|l|}{ AKI } \\
\hline Non-AKI & Reference & & \\
\hline CA-AKI & 2.505 & $1.246-5.035$ & 0.010 \\
\hline HA-AKI & 21.599 & $14.954-31.197$ & $<0.001$ \\
\hline
\end{tabular}

$C K D$ chronic kidney disease; $\mathrm{CCl}$ Charlson comorbidity index; $\mathrm{Hb}$ hemoglobin; $A K I$ acute kidney injury; $C A$ community-acquired; $H A$ hospital-acquired 

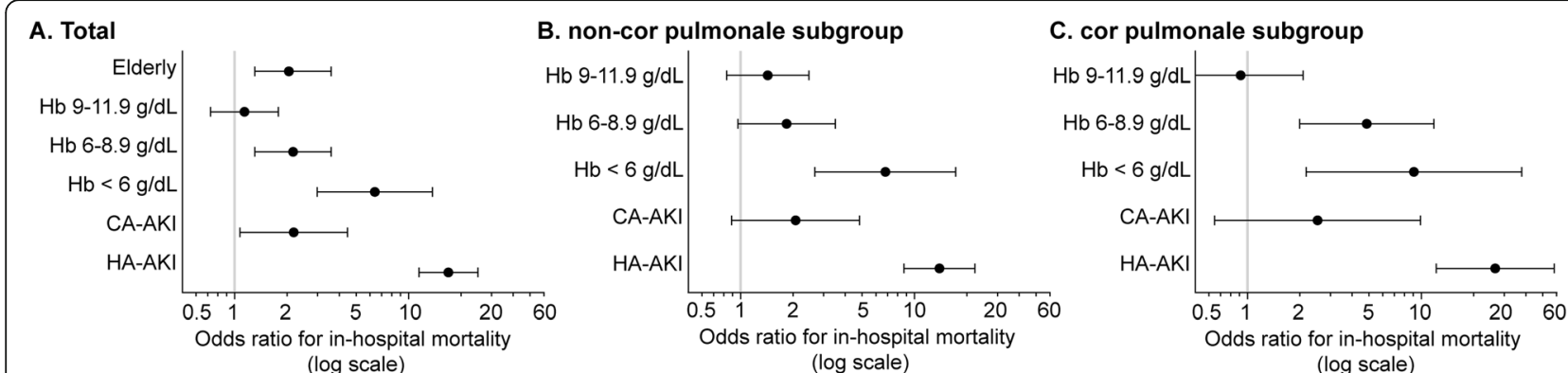

Fig. 2 Association between AKI and in-hospital mortality. The variables included in the multivariate models were increased age (>65 years), female sex, $\mathrm{CCl}$, Hb level (minimum values during hospitalization stratified by $\geq 12,9-11.9,6-8.9 \mathrm{or}<6 \mathrm{~g} / \mathrm{dL}$ ) and AKI. The models included a total of 2897 patients (a), 2236 patients without cor pulmonale (b) and 661 patients with cor pulmonale (c). Hb: hemoglobin; CA-AKl: communityacquired acute kidney injury; HA-AKl: hospital-acquired acute kidney injury

58.176) in the cor pulmonale subgroup]; however, CAAKI was not [OR 2.070 (95\% CI $0.888-4.828)$ in the non-cor pulmonale subgroup; OR 2.532 (95\% CI 0.6479.910) in the cor pulmonale subgroup] (Fig. 2b and c).

\section{Discussion}

In this first epidemiologic study in a hospitalized NECOPD population, we observed that 7.1 and $12.0 \%$ of patients had CA-AKI or HA-AKI, respectively, and the patients with cor pulmonale had a higher incidence of AKI. Increased age, female gender, cor pulmonale comorbidity, CKD stage, diuretic usage, exposure to contrast medium and glycopeptide antibiotic usage before AKI were associated with HA-AKI. After multiple adjustments for age, female gender, $\mathrm{CCI}$ and minimum $\mathrm{Hb}$ level, HA-AKI was an independent risk factor for inhospital mortality, regardless of the presence or absence of cor pulmonale.

Before our study, Cao et al. reported that 15.8 and $5.5 \%$ of hospitalized Chinese patients with AECOPD had CA-AKI and HA-AKI, respectively [7]. The previously reported HA-AKI incidence was lower than that in our study. Theoretically, pathophysiological disturbance is worse in AECOPD than in NECOPD. The inconsistency between the two studies might be attributed to the much longer intervals for baseline $\mathrm{SCr}$ before admission for CA-AKI determination in Cao's study (1 year) than in our study (1 month). An increased interval should cause a lower baseline creatinine level and result in an increased incidence of CA-AKI a reduced incidence of HA-AKI, as in Cao's report. In addition, patients with extremely low $\mathrm{SCr}$ levels were excluded from our study (Fig. 1) but not in Cao's study. Thus, the enrolled patients with HA-AKI in our study had higher creatinine levels (Table 1). Finally, the difference in clinical characteristics between the two studied hospitals might also be involved. In recent epidemiological data, the incidence of total AKI was 11.6\% in hospitalized Chinese patients [11]. Regardless of the AKI type, our and Cao's results together showed that COPD patients had a markedly higher prevalence of AKI than the general hospitalized population. Ours was the first report to reveal the AKI risk in nonexacerbated COPD, which indicates that NECOPD is not a stable condition from a renal perspective.

Previous studies have reported that AECOPD complicated with AKI increased in-hospital mortality $[6,15]$. Our study showed that all types of AKI significantly increased the length of hospital stay and cost in the NECOPD population. After multivariate corrections, complicated HA-AKI remained associated with high inhospital mortality. Although CA-AKI was correlated with an increased risk of in-hospital mortality according to the univariate logistic regression model and the model without the consideration of cor pulmonale, the lower OR limit of HA-AKI was higher than the upper OR limit of CA-AKI, suggesting that the mortality risk in HAAKI patients was significantly higher than that in CAAKI patients. After the patients were subdivided according to the presence of cor pulmonale, no further significant difference was observed. These results agreed with those of a previous study reported by Cao et al. that showed that the risk of in-hospital mortality in HA-AKI patients was significantly higher than that in CA-AKI patients [6].

In this study, all data used for analysis were from the electronic medical records of patients; this strength allowed us to classify the medications administered before AKI. The associations between the use of diuretics, intravenous diodone and glycopeptides and HA-AKI were not surprising; however, the high use prevalence rates above $10 \%$ for diuretics and intravenous diodones should be paid attention to in clinical practice. Another convenience of the use of electronic data was the availability of diagnostic and laboratory records. Thus we could weigh the mortality-related comorbidities, such as cor pulmonale, anemia, CKD and the CCI score. The diagnosis of renal disease is commonly missed in China 
[16]; therefore, we used a complex definition of moderate to severe kidney disease for the CCI score. In addition to the 230 patients diagnosed with moderate to severe kidney disease, 60 patients with an eGFR $<45 \mathrm{ml} /$ $\mathrm{min} / 1.73 \mathrm{~m}^{2}$ were also identified.

Due to the retrospective design, this study failed to differentiate aspirin from other NSAIDs, which might contribute to the negative relationship between the use of NSAIDs, traditionally considered to be drugs with renal toxicity, and HA-AKI. Recent studies have indicated that a low dose of aspirin might protect against or at least not exacerbate AKI $[17,18]$. Therefore, the effects of aspirin and other NSAIDs on AKI needs further evaluation. In addition, our study also had several other limitations. The pulmonary function and blood gas analysis indices were not available. Thus, the severity of hypoxia and the patient conditions could not be evaluated. In addition, approximately one-third of the patients were excluded due to incomplete medical records (Fig. 1), possibly leading to selection bias. Another limitation was that the only adverse outcome observed was in-hospital mortality, and other adverse events, such as quality of life, cardiovascular events and long-term survival, were not investigated.

\section{Conclusion}

AKI is a common condition in the NECOPD population. Diuretics and contrast media are the frequently used medications associated with HA-AKI. Patients with HAAKI have a higher mortality risk than those without AKI.

\section{Abbreviations \\ COPD: Chronic obstructive pulmonary disease; AKI: Acute kidney injury:" NECOPD: Nonexacerbated chronic obstructive pulmonary disease;" CKD: Chronic kidney disease; eGFR: Estimated glomerular filtration rate}

\section{Acknowledgments}

None.

\section{Authors' contributions}

All authors have made contributions to this work and have approved this submission. Conceptualization, XHW and ZX; Methodology, ZX; Software, SGX and WX; Validation, TZ; Formal analysis, WX and TZ; Investigation, SGX and WX; Resources, YS; Data curation, WX and TZ; Writing-original draft preparation, XHW and ZX; Writing-review \& editing, US; Visualization, ZX; Supervision, YS; Project administration, ZX; Funding acquisition, YS.

\section{Funding}

This work was conducted with financial support from the Research Foundation of the Sichuan Provincial Health and Family Planning Commission (17PJ369)

The funder had no role in the design of the study, the collection, analysis and interpretation of the data, or the preparation of the manuscript.

Availability of data and materials

All data used for this analysis were included in the article.

\section{Ethics approval and consent to participate}

The study protocol complied with the Declaration of Helsinki and was approved by the ethics committee of the Sichuan Provincial People's
Hospital (2017-158). Due to the retrospective nature of the study and because the data were analyzed anonymously, the committee waived the requirement for informed consent.

\section{Consent for publication}

Not applicable.

\section{Competing interests}

The authors declare that they have no competing interests.

\section{Author details}

${ }^{1}$ Department of Respiratory Medicine, the Second Affiliated Hospital of Chengdu Medical College, Nuclear Industry 416 Hospital, Chengdu, China. ${ }^{2}$ Department of Dermatology, Sichuan Provincial People's Hospital, University of Electronic Science and Technology of China, Chengdu, China. ${ }^{3}$ Chinese Academy of Sciences Sichuan Translational Medicine Research Hospital, 32 West Second Section, First Ring Road, Chengdu 610031, China. ${ }^{4}$ Division of Urology and Organ Transplantation, Department of Surgery, Sichuan Provincial People's Hospital, University of Electronic Science and Technology of China, Chengdu, China. ${ }^{5}$ Department of Respiratory Medicine, Sichuan Provincial People's Hospital, University of Electronic Science and Technology of China, Chengdu, China. ${ }^{6}$ Clinical Laboratory, Sichuan Provincial People's Hospital, University of Electronic Science and Technology of China, Chengdu, China.

Received: 4 January 2020 Accepted: 20 April 2020

Published online: 29 April 2020

\section{References}

1. Qaseem A, Wilt TJ, Weinberger SE, Hanania NA, Criner G, van der Molen T, et al. Diagnosis and management of stable chronic obstructive pulmonary disease: a clinical practice guideline update from the American College of Physicians, American College of Chest Physicians, American Thoracic Society, and European Respiratory Society. Ann Intern Med. 2011;155:179-91.

2. Gunen H, Hacievliyagil SS, Kosar F, Mutlu LC, Gulbas G, Pehlivan E, et al. Factors affecting survival of hospitalised patients with COPD. Eur Respir J. 2005;26:234-41.

3. Rodríguez A, Lisboa T, Solé-Violán J, Gómez F, Roca O, Trefler S, et al. Impact of nonexacerbated COPD on mortality in critically ill patients. Chest. 2011; 139:1354-60.

4. Husain-Syed F, Slutsky AS, Ronco C. Lung-kidney cross-talk in the critically ill patient. Am J Respir Crit Care Med. 2016;194:402-14.

5. Mehta RL, Cerdá J, Burdmann EA, Tonelli M, García-García G, Jha V, et al. International Society of Nephrology's 0by25 initiative for acute kidney injury (zero preventable deaths by 2025): a human rights case for nephrology. Lancet. 2015;385:2616-43.

6. Fabbian F, De Giorgi A, Manfredini F, Lamberti N, Forcellini S, Storari A, et al. Impact of renal dysfunction on in-hospital mortality of patients with severe chronic obstructive pulmonary disease: a single-center Italian study. Int Urol Nephrol. 2016:48:1121-7.

7. Cao CC, Chen DW, Li J, Ma MQ, Chen YB, Cao YZ, et al. Communityacquired versus hospital-acquired acute kidney injury in patients with acute exacerbation of COPD requiring hospitalization in China. Int J Chron Obstruct Pulmon Dis. 2018;13:2183-90.

8. Kerr M, Bedford M, Matthews B, O'Donoghue D. The economic impact of acute kidney injury in England. Nephrol Dial Transplant. 2014:29:1362-8.

9. Thakar CV, Arrigain S, Worley S, Yared JP, Paganini EP. A clinical score to predict acute renal failure after cardiac surgery. J Am Soc Nephrol. 2005;16:162-8.

10. Kidney Disease: Improving Global Outcomes (KDIGO) Acute Kidney Injury Work Group. KDIGO Clinical Practice Guideline for Acute Kidney Injury. Kid Int Suppl. 2012:1-138.

11. Xu X, Nie S, Liu Z, Chen C, Xu G, Zha Y, et al. Epidemiology and clinical correlates of AKI in Chinese hospitalized adults. Clin J Am Soc Nephrol. 2015:10:1510-8.

12. Kidney Disease: Improving Global Outcomes (KDIGO) CKD Work Group. KDIGO 2012 Clinical Practice Guideline for the Evaluation and Management of Chronic Kidney Disease. Kid Int Suppl. 2013;3:1-150. 
13. Charlson ME, Pompei P, Ales KL, MacKenzie CR. A new method of classifying prognostic comorbidity in longitudinal studies: development and validation. J Chronic Dis. 1987;40:373-83.

14. Sundararajan V, Henderson T, Perry C, Muggivan A, Quan H, Ghali WA. New ICD-10 version of the Charlson comorbidity index predicted in-hospital mortality. J Clin Epidemiol. 2004;57:1288-94.

15. Barakat MF, McDonald HI, Collier TJ, Smeeth L, Nitsch D, Quint JK. Acute kidney injury in stable COPD and at exacerbation. Int J Chron Obstruct Pulmon Dis. 2015;10:2067-77.

16. Zhang L, Wang F, Wang L, Wang W, Liu B, Liu J, et al. Prevalence of chronic kidney disease in China: a cross-sectional survey. Lancet. 2012;379:815-22.

17. Mangano DT. Aspirin and mortality from coronary bypass surgery. N Engl J Med. 2002;347:1309-17.

18. Garg AX, Kurz A, Sessler DI, Cuerden M, Robinson A, Mrkobrada M, et al. Perioperative aspirin and clonidine and risk of acute kidney injury: a randomized clinical trial. JAMA. 2014;312:2254-64.

\section{Publisher's Note}

Springer Nature remains neutral with regard to jurisdictional claims in published maps and institutional affiliations.

Ready to submit your research? Choose BMC and benefit from:

- fast, convenient online submission

- thorough peer review by experienced researchers in your field

- rapid publication on acceptance

- support for research data, including large and complex data types

- gold Open Access which fosters wider collaboration and increased citations

- maximum visibility for your research: over $100 \mathrm{M}$ website views per year

At BMC, research is always in progress.

Learn more biomedcentral.com/submissions 